\title{
Agentes Químicos Ototóxicos (para além dos Solventes)
}

rpso.pt/agentes-quimicos-ototoxicos-para-alem-dos-solventes/

Santos M, Almeida A, Lopes C, Oliveira T. Agentes Químicos Ototóxicos (para além dos Solventes). Revista Portuguesa de Saúde Ocupacional on line. 2020, volume 9, 1-8. DOI: 10.31252/RPSO.23.02.2020

\section{OTOTOXIC CHEMICALS (BEYOND SOLVENTS)}

\author{
TIPO DE ARTIGO: Scoping Review
}

Autores: Santos M(1), Almeida A(2), Lopes C(3), Oliveira T.

\section{RESUMO}

\section{Introdução/ enquadramento/ objetivos}

Classicamente em Saúde Ocupacional associa-se a Hipoacusia à exposição a ruido; contudo, existem vários agentes químicos com essa capacidade. Entre estes, a classe mais frequentemente associada é a dos solventes, ainda que nem sempre com evidência científica irrefutável para alguns casos. Dentro de outras classes de agentes químicos os dados são ainda mais escassos e menos robustos. Pretendeu-se com esta revisão resumir o que de mais recente e pertinente se publicou relativamente a agentes ototóxicos (para além dos solventes).

\section{Metodologia}

Trata-se de uma Revisão iniciada através de uma pesquisa realizada em setembro de 2019 nas bases de dados "CINALH plus with full text, Medline with full text, Database of Abstracts of Reviews of Effects, Cochrane Central Register of Controlled Trials, Cochrane Database of Systematic Reviews, Cochrane Methodology Register, Nursing and Allied Health Collection: comprehensive, MedicLatina, Academic Search Ultimate, Science Direct, Web of Science, SCOPUS e RCAAP”.

\section{Conteúdo}

As classes mais referidas nesse sentido são os solventes (existindo já um artigo publicado nesta revista relacionado com o risco de hipoacusia causada por diversos solventes), metais pesados, agentes asfixiantes e pesticidas. Está também descrito que alguns antibióticos, fármacos anticancerígenos e diuréticos podem ser ototóxicos diretamente. Por sua vez, o cianeto de hidrogénio e o monóxido de carbono podem apresentar efeito sinérgico com o ruído, aliás tal como alguns metais pesados, agentes asfixiantes e pesticidas.

Contudo, em contextos onde existe ruído e agentes químicos ototóxicos, fica difícil perceber o contributo de cada, para além de que pode ocorrer sinergismo. Para além disso, a situação parece estar mais clara em animais; em humanos alguns investigadores assumem que são necessários mais estudos e os níveis a partir dos quais surge lesão não são conhecidos com clareza. Para concentrações mais elevadas a hipoacusia parece evidente mas, para quantidades menores, os dados não são consensuais, por vezes. Também não há consenso quanto à dose mais baixa que terá capacidade de induzir o dano. Para além disso, na maioria das situações ocupacionais a exposição inclui vários agentes químicos e não um só isolado. O metabolismo dos animais não é equivalente ao humano, pelo que as generalizações dos resultados deverão ser feitas com reservas.

A nível laboral, existem menos normas para os agentes químicos, versus ruído, em contexto de perda de audição.

Acredita-se que a ototoxicidade global se possa relacionar com o stress oxidativo; não só devido à formação de espécies reativas de oxigénio, como pela atenuação dos mecanismos de defesa antioxidante.

\section{Discussão, Limitações e Conclusões}

A generalidade dos estudos publicados sobre este tema não é muito robusta, para além de que têm condições heterogéneas entre si; dai que as conclusões destes estudos não se possam generalizar diretamente para a população de trabalhadores expostos a agentes ototóxicos. Ainda assim, entre estes últimos, existem alguns que parecem associar-se de forma mais clara à hipoacusia. Sempre que a associação estiver comprovada e/ ou for suspeita, a equipa de Saúde Ocupacional deverá providenciar medidas que minorem a exposição, de forma a proporcionar o ambiente laboral mais seguro e saudável possível.

Seria relevante conhecer mais sobre a realidade nacional e ver publicados dados de profissionais a exercer em empresas com estes agentes, comparando o efeito de diferentes concentrações/ produtos e/ ou exposição simultânea a ruído (eventualmente a diferentes intensidades), consoante o processo produtivo já existente permitisse. 
PALAVRAS/ EXPRESSÕES- CHAVE: agentes químicos ototóxicos, ototoxicidade, saúde ocupacional e medicina do trabalho.

\section{ABSTRACT \\ Introduction / framework / objectives}

Classically in Occupational Health Hypoacusis is associated to noise exposure; however, there are several chemical agents with this capability. Among these, the most frequently associated are solvents, although not always with irrefutable scientific evidence for some cases. Within other classes of chemical agents the data is even scarcer and less robust. The aim of this review was to summarize the most recent and pertinent publications on ototoxic agents (other than solvents).

\section{Methodology}

This is an Integrative Bibliographic Review, initiated at September 2019 on the databases "CINALH plus with full text, Medline with full text, Database of Abstracts of Reviews of Effects, Cochrane Central Register of Controlled Trials, Cochrane Database of Systematic Reviews, Cochrane Methodology Register, Nursing and Allied Health Collection: Comprehensive, MedicLatina, Academic Search Ultimate, Science Direct, Web of Science, SCOPUS and RCAAP".

\section{Content}

The most commonly mentioned classes are solvents (there is already an article published in this journal related to the risk of hypoacusis), heavy metals, asphyxiants and pesticides. It is also described that some antibiotics, anticancer drugs and diuretics may be ototoxic directly. In turn, hydrogen cyanide and carbon monoxide can have a synergistic effect with noise, as well as some heavy metals, asphyxiants and pesticides.

However, in contexts where there is noise and ototoxic chemical agents, it is difficult to understand the contribution of each, and synergism may occur. Moreover, the situation seems to be clearer in animals; In humans some researchers assume that further studies are needed and the levels at which injury arises are not known clearly. At higher concentrations, hypoacusis seems evident, but for smaller amounts, data are not always consensual. There is also no consensus on the lowest dose that will induce damage. In addition, in most occupational situations exposure includes several chemical agents and not one isolated. Animal is not equivalent to human metabolism, so generalizations of results should be made with reservations.

At work level, there are fewer standards for chemical versus noise agents in the context of hearing loss.

Global ototoxicity is believed to be related to oxidative stress; not only due to the formation of reactive oxygen species, but also through the attenuation of antioxidant defense mechanisms.

\section{Discussion, Limitations, and Conclusions}

Most of the published studies on this subject are not very robust, besides that they have heterogeneous conditions among themselves. Hence, the conclusions of these studies cannot be generalized directly to the population of workers exposed to ototoxic agents. Still, among the latter there are some that seem to associate more clearly with hearing loss. Whenever the association is proven and/ or suspected, the Occupational Health team should provide measures that mitigate exposure to provide the safest and healthiest working environment possible.

It would be relevant to know more about the national reality and to have published data of professionals working in companies with these agents, comparing the effect of different concentrations/ products and/ or simultaneous exposure to noise (possibly at different intensities), depending on the existing production process.

KEY WORDS / EXPRESSIONS: ototoxic chemical agents, ototoxicity, occupational health and occupational medicine.

\section{INTRODUÇÃO}

Na Saúde Ocupacional classicamente associa-se a hipoacusia à exposição a ruido; contudo, existem vários agentes químicos com essa capacidade. Entre estes, a classe mais frequentemente associada é a dos solventes, ainda que nem sempre com evidência científica irrefutável para alguns casos, tendo esta revista já publicado outro artigo, no ano de 2019, na qual abordou essa temática (Solventes e Hipoacusia- qual a evidência?). Dentro de outras classes de agentes químicos, os dados são ainda mais escassos e menos robustos.

Pretendeu-se com esta revisão resumir o que de mais recente e pertinente se publicou relativamente a agentes ototóxicos (para além dos solventes).

\section{METODOLOGIA}

Pergunta protocolar: quais os agentes químicos com propriedades ototóxicas, para além dos solventes?

Em função da metodologia PICo, foram considerados: 
-P (population): trabalhadores que utilizam agentes químicos ototóxicos, para além dos solventes.

-I (interest): reunir conhecimentos relevantes sobre ototoxicidade por agentes químicos, para além dos solventes.

-C (context): saúde ocupacional nas empresas com postos de trabalho com agentes ototóxicos, para além dos solventes.

Foi realizada uma pesquisa em setembro de 2019 nas bases de dados "CINALH plus with full text, Medline with full text, Database of Abstracts of Reviews of Effects, Cochrane Central Register of Controlled Trials, Cochrane Database of Systematic Reviews, Cochrane Methodology Register, Nursing and Allied Health Collection: comprehensive, MedicLatina, Academic Search Ultimate, Science Direct, Web of Science, SCOPUS e RCAAP".

No quadro 1 podem ser consultadas as palavras/ expressões- chave utilizadas nas bases de dados.

\section{CONTEÚDO}

\section{Audição, Ruído e Hipoacusia}

O ouvido tem uma área externa, média e interna. A primeira é constituída pelo pavilhão auricular e canal auditivo externo. Por sua vez, o ouvido médio é formado pelo tímpano, martelo, bigorna e estribo. O ouvido interno inclui as células ciliadas internas e externas, bem como a cóclea. A audição é um sentido complexo, que implica a capacidade que o ouvido tem para detetar sons e do cérebro, para os interpretar. A perda de audição pode justificar-se com a exposição ao ruído, envelhecimento, doenças específicas e exposição a alguns agentes químicos (nomeadamente em contexto laboral) [1]. Geralmente considera-se que há perda auditiva quando os valores obtidos são inferiores em, pelo menos, 25 decibéis (dBs), nas frequências de 1000, 2000, 3000 e 4000 Hzs [2]. A exposição ao ruído constitui uma etiologia consensualmente aceite de hipoacusia [3]. Aliás, este é considerado como a principal justificação para a perda auditiva ocupacional [4]. A diminuição da audição pode ser mais rápida ou mais gradual e a gravidade relaciona-se com a quantidade, tempo de exposição e interação com outros agentes físicos ou químicos [1]. A perda de audição pode contribuir para o isolamento social e sinistralidade laboral [5].

\section{Agentes químicos em geral e Ototoxicidade}

Como pode ser complicado atenuar os níveis de ruído em alguns setores profissionais, torna-se pertinente identificar e também suprimir (ou, pelo menos, atenuar) outros fatores de risco que possam estar presentes e que contribuam para a hipoacusia [3], nomeadamente alguns agentes químicos, classificados como ototóxicos. As classes mais referidas nesse sentido são os solventes [3] [6] [7] [8], metais pesados [3] [4] [7] [9], agentes asfixiantes [3] [6] [7] [9] e pesticidas [3] [7] [9]. Está também descrito que alguns antibióticos, fármacos anticancerígenos e diuréticos podem ser ototóxicos diretamente. Por sua vez, o cianeto de hidrogénio [8] e o monóxido de carbono podem apresentar efeito sinérgico com o ruido [6] [8], aliás tal como alguns metais pesados, agentes asfixiantes e pesticidas [3].

Contudo, em contextos onde existe ruído e agentes químicos ototóxicos, fica difícil perceber o contributo de cada, para além de que pode ocorrer sinergismo [3]. A situação parece estar mais clara em animais; em humanos alguns investigadores assumem que são necessários mais estudos e os níveis a partir dos quais surge lesão não são conhecidos com clareza [7]. Para concentrações mais elevadas a hipoacusia parece evidente mas, para quantidades menores, os dados não são consensuais, por vezes. Também não há concordância quanto à dose mais baixa que terá capacidade de induzir o dano [6]. Para além disso, na maioria das situações ocupacionais a exposição inclui vários agentes químicos e não um só isolado [3] [6]. O metabolismo dos animais não é equivalente ao humano, pelo que as generalizações dos resultados deverão ser feitas com reservas [6].

A nível laboral, existem menos normas para os agentes químicos, versus ruído, em contexto de perda de audição [3].

Acredita-se que a ototoxicidade global se possa relacionar com o stress oxidativo; não só devido à formação de espécies reativas de oxigénio, como pela atenuação dos mecanismos de defesa antioxidante [5].

\section{Metais pesados em geral e Ototoxicidade}

Alguns investigadores estimaram um aumento de risco de hipoacusia na ordem de 1,64 vezes para metais pesados, globalmente. Estes podem causar apoptose e/ ou degeneração das células do ouvido interno, originando disfunções audiológicas neuronais. Os metais pesados participam no controlo do equilíbrio intracelular, logo, com capacidade para causar danos nas células ciliadas. Ainda assim, o mecanismo exato não é conhecido na totalidade [3]. A hipoacusia é mais percetível a nível das frequências mais elevadas [3]. Alguns investigadores destacam neste contexto o cádmio, chumbo, mercúrio [3] [10] e manganésio [3].

Outros realçam que existe evidência clara de que o chumbo é ototóxico [5] [6] [8], quer em animais, quer humanos [6] [10], eventualmente por indução da apoptose ou degeneração do ouvido interno através das alterações na homeostasia do cálcio [3] e/ ou através da alteração na permeabilidade da barreira entre a cóclea e a circulação sanguínea, depositando-se no ouvido interno [11]. Contudo, a perda de audição associada ao chumbo apresenta evidências contraditórias [10] [11]: alguns estudos concluíram que existe diminuição da audição para exposições intensas e crónicas, mas não para exposições mais curtas e há quem considere que não há consenso perante o eventual sinergismo com o ruído [10]. Beethoven, por exemplo, apresentava uma deficiência auditiva grave. Na sua autópsia não foram encontradas evidências de otosclerose; contudo, constatou-se um nível elevado de chumbo ósseo, indicativo de exposição crónica (provavelmente associado ao consumo de vinho com esse agente); a perda de audição nele foi progressiva ao longo dos anos [12]. O chumbo associa-se a perdas auditivas entre os 3000 e $8000 \mathrm{Hzs}$, de forma estatisticamente significativa (mesmo aplicando a técnica da regressão logística, de forma a controlar eventuais variáveis enviesadoras), ainda que o mecanismo exato, segundo alguns investigadores, não esteja claramente definido [13]. 
O cádmio pode originar perda auditiva em ratos e pode apresentar sinergismo com o ruido; o efeito parece ser dose-dependente e através da acumulação na proximidade dos ossículos e nas células de cóclea [10]. Outros afirmam que o mecanismo não é conhecido com clareza, mas supõe-se que se possa relacionar com a alteração na proliferação e diferenciação celular, síntese e metilação do DNA e apoptose, originando desequilíbrios em algumas proteínas [11].

O arsénio poderá originar perda de audição para baixas e altas frequências, ainda que a suscetibilidade entre indivíduos varie [10].

O manganésio em quantidades elevadas e com exposição crónica, poderá induzir perda de audição em animais e humanos, ainda que alguns defendam que tal facto poderá ser influenciado por variáveis confundidoras, como ruído, idade e tabagismo [11].

O mercúrio pode diminuir a audição [5] [9] [11] devido a danos periféricos e centrais [11].

A administração simultânea de crómio e cobalto em coelhos originou alterações auditivas, baseadas sobretudo em danos cocleares que parecem ser dose-dependentes [14].

O cobalto [9] [11], quer via inalatória em contexto ocupacional, quer absorvido através de implantes médicos metálicos, poderá originar perda de audição. Existem poucos estudos em animais e as conclusões existentes para humanos são provenientes parcialmente dos danos observados em implantes médicos desta substância, com algum defeito [11].

\section{Pesticidas}

Os pesticidas poderão contribuir para a perda de audição ocupacional [15] [16] [17] [18]; aliás, a hipoacusia poderá constituir uma evidência de intoxicação- trabalhadores que usam pesticidas com alguma regularidade apresentam alterações auditivas [19]. Os danos incidem sobretudo na cóclea e no nervo auditivo, induzindo alterações vestibulares e no sistema nervoso central [15]; ainda que, simultaneamente, alguns autores recomendem estudos mais aprofundados relativamente à ototoxicidade dos pesticidas [5]. Tal efeito também está comprovado em animais [19]. Alguns, dentro desta classe, destacam os organofosforados [16]; estes podem potenciar a formação de espécies reativas de oxigénio e apresentar sinergismo com o ruído [19].

\section{DISCUSSÃO/ LIMITAÇÕES/ CONCLUSÃO}

A generalidade dos estudos publicados sobre este tema não é muito robusta, para além de que têm condições heterogéneas entre si (investigação em animais versus humanos, amostras de tamanho diverso, incluir observações com e sem ruido- com intensidades diferentes ou sem quantificação de tal, concentrações de agentes químicos diversas e/ ou ausência de menção à concentração, tempos de exposição diversos ou ausência de referência dos mesmos); dai que as conclusões destes estudos não se possa generalizar diretamente para a população de trabalhadores expostos a agentes ototóxicos. Ainda assim, entre estes últimos, existem alguns que parecem associar-se de forma mais clara à hipoacusia. Sempre que a associação estiver comprovada e/ ou for suspeita, a equipa de Saúde Ocupacional deverá providenciar medidas que minorem a exposição, de forma a proporcionar o ambiente laboral mais seguro e saudável possível.

Seria relevante conhecer mais sobre a realidade nacional e ver publicados dados de profissionais a exercer em empresas com estes agentes, comparando o efeito de diferentes concentrações/ produtos e/ ou exposição simultânea a ruido (eventualmente a diferentes intensidades), consoante o processo produtivo já existente permitisse.

\section{CONFLITOS DE INTERESSE, QUESTÕES ÉTICAS E/OU LEGAIS}

Nada a declarar.

\section{AGRADECIMENTOS}

Nada a declarar.

\section{BIBLIOGRAFIA}

[1] Pleban F, Shrestha L, Oketope O, Pleban S. Xylene exposure on auditory function among adults in selected occupational setting: a systematic review. Taiwan Journal of Public Health. 2017, 36(39, 204-214.

[2] Hormozi M, Ansari-Moghaddam A, Mirzali R, Haghighi J, Eftekharian f. The risk of hearing loss associated with occupational exposure to organic solvents mixture with and without concurrent noise exposure: a systematic review and meta-analysis. International Journal of Occupational Medicine and Environmental Health. 2017, 30(4), 521-535. DOI: 10.13075/jomeg.01024

[3] Choi Y, Kim K. Noise- induced hearing loss in korean workers: co-exposure to organic solventes and heavy metals in nationwide industries. PLOS ONE. 2014, 9(5), e97538, 1-9. DOI: 10.1371/journal.pone0097538

[4] Metwally F, Aziz H, Mahdy-Abdallah H, Elgelil K, El-Tahlawy E. Effect of combined occupational exposure to noise and organic solvents on hearing. Toxicology and Industrial Health. 2014, 28(10), 901-907. DOI: 10.1177/0748233711427051

[5] Gatto M, Fioertti M, Fabrizi G, Gherardi M, Strafella E, Santarelli L. Effects of potencial neurotoxic pesticides on hearing loss: a review. Neurotoxicology. 2014, 42, 24-32. DOI: 10.1016/j.neuro.2014.03.009 
[6] Vyskocil A, Truchon G, Leroux T, Lemay F, Gendron M, Gagnon F et al. A Weight of evidence approach for the assessment of the ototoxic potential of industrial chemicals. Toxicology and Industrial Health. 2012, 28(9), 796-819. DOI: $10.1177 / 0748223711425067$

[7] Hammill T, McKenna E, Hecht Q, Buchanan K, Pryor N. I'm wearing Hearing Protection- Am I still at risk for Hearing Loss? Lurking ototoxins in the Military Environment. Military Medicine. 2019, 184, 3/4, 615-620.

[8] Autoridade para as Condições do Trabalho. Guia indicativo de Boas Práticas para a aplicação da Diretiva 2003/ 10/CE "Ruído no Trabalho". Comissão Europeia. direção Geral do emprego, Assuntos Sociais e lgualdade de Oportunidades. 2007, 1-175.

[9] Botelho C, Lopes A, Gonçalves A, Frota S. Comparative study of audiometries tests on metallurgical workers exposed to noise only as well as noise associated to the handling of chemical products. Brazilian Journal of Otorhinolaryngology. 2009, 75(1), 51-57.

[10] Kang G, Uhm J, Choi Y, Kang E, Kim S, Choo W et al. Environmental exposure to heavy metal (lead and cadmium) and hearing loss: data from the Korea National Health and Nutrition Examination Survey (KNH ANES 2010-2013). Annals of Occupational and Environmental Medicine. 2018, 30(22), 1-11. DOI: 1186/s40557-018-0237-9

[11] Roth J, Salvi R. Ototoxicity of divalente metals. Neurotoxicity Research. 2016, 30, 268-282. DOI: 10.1007/s12640-016-9627-3

[12] Stevens M, Jacobsen T, Croft A. Lead and the Deafness of Ludwig Van Beethoven. The Laryngoscope. 2013, 123, 28542858.

[13] Hwang $Y$, Chiang $\mathrm{H}$, Yen-Jean M, Wang J. The association between low levels of lead in blood and occupational noiseinduced hearing loss in stell workers. Science of the Total Environment. 2009, 408, 43-49.

[14] Apostoli P, Catalani S, Zaghini A, Mariotti A, Poliani P, Vielmi V et al. High doses of cobalt induce optic and auditory neuropathy. Experimental and Toxicologic Pathology. 2013, 65, 719-727. DOI: 10.1016/j.etp.2012.09.006

[15] Sena T, Dourado S, Lima L, Antoniolli A. The hearing of rural workers exposed to noise and pesticides. Noise \& Health. 2018, 20(92), 23-26.

[16] Silver M, Shao J, Ji C, Zhu B, Xu L, Li M et al. Prenatal organophosphate insecticide exposure and infant sensory function. International Journal of Hygiene and Environmental Health. 2018, 221. 469-478. DOI: 10.1016/j.ijheh.2018.01.010

[17] Kos M, Hoshino A, Asmus C, Mendonça R, Meyer A. Efeitos da exposição a agrotóxicos sobre o sistema auditivo periférico e central: uma revisão sistemática. Cadernos de Saúde Pública. 2013, 29(8), 1491-1505. DOI: 10.1590/0102-311×0007013

[18] Bazílio M, Frota S, Chrisman J, Meyer A, Asmus C, Camara V. Processamento auditivo temporal de trabalhadores rurais expostos a agrotóxico. Jornal da Sociedade Brasileira de Fonoaudiologia. 2012, 24(2), 174-180.

[19] França D, Lacerda A, Lobato D, Ribas A, Dias K, Leroux T et al. Adverse effects of pestivides on central auditory functions in tobacco growers. International Journal of Audiology. 2016, 56(4), 233-241. DOI: 10.1080/14992027-2016.1255787

Quadro 1: Pesquisa efetuada

\begin{tabular}{|c|c|c|c|c|c|c|c|c|c|}
\hline $\begin{array}{l}\text { Motor de } \\
\text { busca }\end{array}$ & $\begin{array}{l}\text { Password } \\
1\end{array}$ & $\begin{array}{l}\text { Password } \\
2 \text { e } \\
\text { seguintes, } \\
\text { caso } \\
\text { existam }\end{array}$ & Critérios & $\begin{array}{l}\mathrm{N}^{\circ} \text { de } \\
\text { documentos } \\
\text { obtidos }\end{array}$ & $\begin{array}{l}N^{\circ} \text { da } \\
\text { pesquisa }\end{array}$ & $\begin{array}{l}\text { Pesquisa } \\
\text { efetuada } \\
\text { ou não }\end{array}$ & $\begin{array}{l}N^{\circ} \text { do } \\
\text { documento } \\
\text { na } \\
\text { pesquisa }\end{array}$ & $\begin{array}{l}\text { Codificação } \\
\text { inicial }\end{array}$ & $\begin{array}{l}\text { Codificação } \\
\text { final }\end{array}$ \\
\hline
\end{tabular}

(documentos utilizados no artigo relativo à hipoacusia e solventes, que também mencionavam outros agentes químicos ototóxicos)

$\begin{array}{ll}- & 1 \\ - & 2 \\ - & 3 \\ - & 4 \\ - & 6 \\ - & 8\end{array}$

\begin{tabular}{|c|c|c|c|c|c|c|c|c|c|}
\hline \multirow{5}{*}{$\begin{array}{l}\text { Academic } \\
\text { Search } \\
\text { Ultimate }\end{array}$} & Hypoacusis & & \multirow{3}{*}{$\begin{array}{l}-2009 \text { a } \\
2019 \\
\text {-texto } \\
\text { completo } \\
\text {-humano }\end{array}$} & 81 & 1 & Sim & & & \\
\hline & \multirow[t]{4}{*}{ Deafness } & \multirow[b]{2}{*}{ Asphyxiants } & & 13.387 & 2 & Não & & & \\
\hline & & & & 2 & 3 & Sim & $\begin{array}{l}1 \\
2\end{array}$ & $\begin{array}{l}3.1 \\
3.2\end{array}$ & $\begin{array}{l}7 \\
5\end{array}$ \\
\hline & & Pesticides & & 4 & 4 & Sim & $\begin{array}{l}1 \\
2\end{array}$ & $\begin{array}{l}4.1 \\
4.2\end{array}$ & $\begin{array}{l}15 \\
-\end{array}$ \\
\hline & & & & & & & 4 & 4.3 & 16 \\
\hline
\end{tabular}




Heavy
metals

$$
7
$$$$
5
$$

Sim

1
2

$=3$

5.2

-

$3 \quad 5.3$

4

5.4

6

5.5

11

\begin{tabular}{|c|c|c|c|c|c|c|c|c|c|}
\hline \multirow{9}{*}{$\begin{array}{l}\text { Science } \\
\text { Direct }\end{array}$} & \multirow[t]{4}{*}{ Hypoacusis } & & & 455 & 6 & Não & & & \\
\hline & & Asphyxiants & & 2 & 7 & Sim & 1 & 7.1 & 9 \\
\hline & & Pesticides & & 3 & 8 & Sim & & & \\
\hline & & $\begin{array}{l}\text { Heavy } \\
\text { metals }\end{array}$ & & 9 & 9 & Sim & & & \\
\hline & \multirow[t]{5}{*}{ Deafness } & & & 21.687 & 10 & Não & & & \\
\hline & & Asphyxiants & & 20 & 11 & Sim & & & \\
\hline & & Pesticides & & 345 & 12 & Não & & & \\
\hline & & $\begin{array}{l}\text { Heavy } \\
\text { metals }\end{array}$ & & 854 & 13 & Não & & & \\
\hline & & $\begin{array}{l}\text { Pesticides+ } \\
\text { Heavy } \\
\text { metals }\end{array}$ & & 169 & 14 & Sim & Sem $n^{\circ}$ & $=5$ & - \\
\hline \multirow[t]{10}{*}{ SCOPUS } & \multirow[t]{4}{*}{ Hypoacusis } & & & 323 & 15 & Não & & & \\
\hline & & Asphyxiants & & 0 & 16 & Não & & & \\
\hline & & Pesticides & & 0 & 17 & Não & & & \\
\hline & & $\begin{array}{l}\text { Heavy } \\
\text { metals }\end{array}$ & & 2 & 18 & Sim & & & \\
\hline & \multirow[t]{6}{*}{ Deafness } & & & 21.212 & 19 & Não & & & \\
\hline & & Asphyxiants & & 1 & 20 & Sim & 1 & $=3.2$ & \\
\hline & & Pesticides & & 5 & 21 & Sim & $\begin{array}{l}2 \\
3\end{array}$ & $\begin{array}{l}21.1 \\
21.2\end{array}$ & $\begin{array}{l}19 \\
17\end{array}$ \\
\hline & & & & & & & 4 & 21.3 & 18 \\
\hline & & $\begin{array}{l}\text { Heavy } \\
\text { metals }\end{array}$ & & 17 & 22 & Sim & $\begin{array}{l}10 \\
11\end{array}$ & $\begin{array}{l}22.1 \\
22.2\end{array}$ & $\begin{array}{l}12 \\
13\end{array}$ \\
\hline & & & & & & & 16 & 22.3 & 14 \\
\hline \multirow{5}{*}{$\begin{array}{l}\text { Web of } \\
\text { Science }\end{array}$} & \multicolumn{2}{|l|}{ Hypoacusis } & & 104 & 23 & Sim & & & \\
\hline & \multirow[t]{4}{*}{ Deafness } & & & 9.588 & 24 & Não & & & \\
\hline & & Asphyxiants & & 0 & 25 & Não & & & \\
\hline & & Pesticides & & 1 & 26 & Sim & & & \\
\hline & & $\begin{array}{l}\text { Heavy } \\
\text { metals }\end{array}$ & & 4 & 27 & Sim & & $=22.1$ & \\
\hline \multirow[t]{5}{*}{ EBSCO } & Hypoacusis & & & 34 & 28 & Sim & & & \\
\hline & \multirow[t]{4}{*}{ Deafness } & & & 4.599 & 29 & Não & & & \\
\hline & & Asphyxiants & & 0 & 30 & Não & & & \\
\hline & & Pesticides & & 1 & 31 & Sim & 1 & $=5.5$ & \\
\hline & & $\begin{array}{l}\text { Heavy } \\
\text { metals }\end{array}$ & & 1 & 32 & Sim & & & \\
\hline \multirow[t]{3}{*}{ RCAAP } & Hipoacusia & & \multirow{3}{*}{$\begin{array}{l}\text {-pesquisa } \\
\text { - avançada } \\
\text {-título }\end{array}$} & 44 & 33 & Sim & & & \\
\hline & Surdez & & & 293 & 34 & Não & & & \\
\hline & & Asfixiantes & & 0 & 35 & Não & & & \\
\hline
\end{tabular}




\begin{tabular}{|c|c|c|c|}
\hline Pesticidas & 0 & 36 & Não \\
\hline $\begin{array}{l}\text { Metais } \\
\text { pesados }\end{array}$ & 0 & 37 & Não \\
\hline
\end{tabular}

(1)Mónica Santos

Licenciada em Medicina; Especialista em Medicina Geral e Familiar; Mestre em Ciências do Desporto; Especialista em Medicina do Trabalho e Doutoranda em Segurança e Saúde Ocupacionais, na Faculdade de Engenharia da Universidade do Porto. Presentemente a exercer nas empresas Medicisforma, Servinecra, Securilabor e Medimarco; Diretora Clínica da empresa Quercia; Diretora da Revista Portuguesa de Saúde Ocupacional on line. Endereços para correspondência: Rua Agostinho Fernando Oliveira Guedes, 42, 4420-009 Gondomar. E-mail: s_monica_santos@hotmail.com

\section{(2)Armando Almeida}

Enfermeiro Especialista em Enfermagem Comunitária, com Competência Acrescida em Enfermagem do Trabalho. Doutorado em Enfermagem; Mestre em Enfermagem Avançada; Pós-graduado em Supervisão Clínica e em Sistemas de Informação em Enfermagem; Professor Auxiliar Convidado na Universidade Católica Portuguesa, Instituto da Ciências da Saúde - Escola de Enfermagem (Porto) onde Coordena a Pós-Graduação em Enfermagem do Trabalho; Diretor Adjunto da Revista Portuguesa de Saúde Ocupacional on line. 4420-009 Gondomar. E-mail: aalmeida@porto.ucp.pt

\section{(3)Catarina Lopes}

Licenciada em Enfermagem, desde 2010, pela Escola Superior de Saúde Vale do Ave. A exercer funções na área da Saúde Ocupacional desde 2011 como Enfermeira do trabalho autorizada pela Direção Geral de Saúde, tendo sido a responsável pela gestão do departamento de Saúde Ocupacional de uma empresa prestadora de serviços externos durante 7 anos. Atualmente acumula funções como Enfermeira de Saúde Ocupacional e exerce como Enfermeira Generalista na SNS24. Encontra-se a frequentar o curso Técnico Superior de Segurança do Trabalho. 4715-028. Braga. E-mail: catarinafflopes@gmail.com

\section{(4)Tiago Oliveira}

Licenciado em Enfermagem pela Universidade Católica Portuguesa. Frequenta o curso de Técnico Superior de Segurança no Trabalho. Atualmente exerce a tempo inteiro como Enfermeiro do Trabalho. No âmbito desportivo desenvolveu competências no exercício de funções de Coordenador Comercial na empresa Academia Fitness Center, assim como de Enfermeiro pelo clube de futebol União Desportiva Valonguense. 4435-718 Baguim do Monte. E-mail: tiago_sc16@hotmail.com 